\title{
Musicianship and melodic predictability enhance neural gain in auditory cortex during pitch deviance detection
}

\author{
David R. Quiroga-Martinez $^{1}$ ( ) | Niels Christian Hansen ${ }^{1,2}$ | Andreas Højlund ${ }^{3}$ | \\ Marcus Pearce $^{4}$ | Elvira Brattico ${ }^{1,5}$ | Emma Holmes ${ }^{6}$ | Karl Friston ${ }^{6}$ ¿ $_{\text {| }}$ \\ Peter Vuust $^{1}$
}

${ }^{1}$ Center for Music in the Brain, Aarhus University \& Royal Academy of Music Aarhus/ Aalborg, Aarhus, Denmark

${ }^{2}$ Aarhus Institute of Advanced Studies, Aarhus University, Aarhus, Denmark

${ }^{3}$ Center for Functionally Integrative Neuroscience, Aarhus University, Aarhus, Denmark

${ }^{4}$ School of Electronic Engineering and Computer Science, Queen Mary University of London, London, UK

${ }^{5}$ Department of Education, Psychology and Communication, University of Bari Aldo Moro, Bari, Italy

${ }^{6}$ The Wellcome Centre for Human Neuroimaging, UCL Queen Square Institute of Neurology, UCL, London, UK

\section{Correspondence}

David R. Quiroga-Martinez, Center for Music in the Brain, Aarhus University \& Royal Academy of Music Aarhus/Aalborg, Aarhus, Denmark.

Email: dquiroga@clin.au.dk

Funding information

Action on Hearing Loss, Grant/Award Number: PA_25; Danmarks Grundforskningsfond, Grant/Award Number: DNRF 117; H2020 Marie Skłodowska-Curie Actions, Grant/Award Number: 754513; Wellcome Trust, Grant/ Award Number: 088130/Z/09/Z

\begin{abstract}
When listening to music, pitch deviations are more salient and elicit stronger prediction error responses when the melodic context is predictable and when the listener is a musician. Yet, the neuronal dynamics and changes in connectivity underlying such effects remain unclear. Here, we employed dynamic causal modeling (DCM) to investigate whether the magnetic mismatch negativity response ( $\mathrm{MMNm}$ )-and its modulation by context predictability and musical expertise-are associated with enhanced neural gain of auditory areas, as a plausible mechanism for encoding precision-weighted prediction errors. Using Bayesian model comparison, we asked whether models with intrinsic connections within primary auditory cortex (A1) and superior temporal gyrus (STG)-typically related to gain control-or extrinsic connections between A1 and STG-typically related to propagation of prediction and error signals-better explained magnetoencephalography responses. We found that, compared to regular sounds, outof-tune pitch deviations were associated with lower intrinsic (inhibitory) connectivity in A1 and STG, and lower backward (inhibitory) connectivity from STG to A1, consistent with disinhibition and enhanced neural gain in these auditory areas. More predictable melodies were associated with disinhibition in right $\mathrm{A} 1$, while musicianship was associated with disinhibition in left A1 and reduced connectivity from STG to left A1. These results indicate that musicianship and melodic predictability, as well as pitch deviations themselves, enhance neural gain in auditory cortex during deviance detection. Our findings are consistent with predictive processing theories suggesting that precise and informative error signals are selected by the brain for subsequent hierarchical processing.

KEYWORDS

DCM, MMN, musical expertise, neural gain, precision, salience
\end{abstract}

\section{1 | INTRODUCTION}

Surprising sounds in auditory sequences are perceived as more salient and generate stronger neural responses than expected sounds
(Heilbron \& Chait, 2018). The salience of surprising sounds and the responses they elicit depend on at least two factors: the musical expertise of the listener and the predictability of the auditory context. In music, for example, pitch deviants elicit a mismatch response-the 
mismatch negativity ( $\mathrm{MMN}$ )-taken to reflect the update of the brain's internal predictive model by prediction error (Vuust, Brattico, Seppänen, Näätänen, \& Tervaniemi, 2012). Crucially, deviants are more easily detected and the amplitude of the MMN becomes larger, when melodies are predictable and when the listener is a musician (QuirogaMartinez et al., 2019b). Yet, despite the growing empirical evidence (Garrido, Sahani, \& Dolan, 2013; Hsu, Bars, Hämäläinen, \& Waszak, 2015; Kliuchko et al., 2019; Southwell \& Chait, 2018; Tervaniemi, Huotilainen, \& Brattico, 2014), little is known about the fluctuations in effective connectivity and neuronal dynamics that mediate these phenomena.

The effect of context predictability on sound salience has been proposed to reflect the neural weighting of unexpected events by the precision afforded to sensory inputs (Quiroga-Martinez et al., 2019a; Ross \& Hansen, 2016; Vuust, Dietz, Witek, \& Kringelbach, 2018). Such a precision-weighting mechanism would allow the brain to select informative sensory signals for further processing (Feldman \& Friston, 2010; Friston et al., 2020; Hohwy, 2012). Research on attention has linked this selection to a modulation of postsynaptic gain in which the activity of neurons representing attended features and objects is enhanced (Garrido, Rowe, Halász, \& Mattingley, 2018; Rabinowitz, Goris, Cohen, \& Simoncelli, 2015; Reynolds \& Desimone, 1999). Such gain modulation likely arises from a change in the strength of intrinsic (i.e., within-region) connections controlling the excitability of brain areas (Auksztulewicz et al., 2017, 2018; Auksztulewicz \& Friston, 2015), usually ascribed to NMDA receptor function and synchronous interactions between fast-spiking inhibitory interneurons and pyramidal cells. However, it remains unclear whether the same gain mechanisms operate when the salience of a sound is driven, not endogenously by selective attention, but rather exogenously by the predictability of successive stimuli.

The effect of musicianship on sound salience has also been suggested to rely on precision-driven mechanisms. Vuust et al. (2018) proposed that musicians possess a more precise predictive model of musical auditory signals than nonmusicians, a view that has behavioral support (Hansen \& Pearce, 2014; Hansen, Vuust, \& Pearce, 2016). If musicians have a fine-grained representation of musical tuning-which facilitates deviance detection and leads to enhanced MMN responses-the same precision-driven gain mechanisms above may also operate when sound salience is enhanced by musical expertise.

Here, we characterized the neuronal dynamics and effective connectivity underlying the salience of surprising musical sounds and its modulation by predictability and musical expertise. We employed dynamic causal modeling (DCM) of magnetoencephalography (MEG) data from a previous study investigating magnetic MMN responses (MMNm) in melodic sequences (Quiroga-Martinez et al., 2019b). In that experiment, musicians and nonmusicians listened to highly predictable stimuli-a repeated four-note pattern-and less predictable stimuli-complex, less-repetitive melodies. We found that pitch deviants were more easily detected and generated larger $\mathrm{MMNm}$ responses in highly predictable compared to less predictable melodies, and in musicians compared to nonmusicians.
We based the DCM analyses on an auditory network comprising bilateral primary auditory cortex (A1), bilateral superior temporal gyrus (STG), and the right frontal operculum (rFOP). We asked whether the MMNm, and its modulation by predictability and musical expertise, relied on changes in intrinsic connectivity within A1 and STG, as a plausible synaptic mechanism implementing precision-weighting of prediction error. We compared this to the alternative explanation that predictability and expertise modulate propagation of prediction and error signals through forward and backward extrinsic (i.e., betweenregions) connectivity, which is typically associated with short-term plasticity, sensory learning and model updating. Thus, we contrasted models in which intrinsic, forward, and/or backward connections were allowed to explain auditory evoked responses and their modulation as measured with MEG.

\section{2 | METHODS}

Reproducible code and data are available at https://doi.org/10. 17605/osf.io/bdr73.

\section{1 | Participants}

Twenty musicians and 20 nonmusicians were included in the study. These participants are part of a larger group of 24 nonmusicians and 26 musicians whose data have been analyzed and reported elsewhere (Quiroga-Martinez, et al., 2019a, 2019b; Quiroga-Martinez, et al., 2020). The four nonmusicians and six musicians excluded were those for whom high-quality MRI images were not available, due to artifacts or abstaining from the MRI session. Musical expertise and musical competence (Table 1) were assessed with the Goldsmiths Musical Sophistication Index (GMSI) (Müllensiefen, Gingras, Musil, \& Stewart, 2014) and the Musical Ear Test (MET) (Wallentin, Højlund, Friis-Olivarius, Vuust, \& Vuust, 2010). Musicians had significantly higher GMSI $(t[31.1]=14.82, p<.001)$ and were significantly better at differentiating melodies/rhythms than nonmusicians, as indicated by the total scores on the MET $(t[35.2]=5.38, p<.001)$. See QuirogaMartinez, et al. (2020) and Quiroga-Martinez, et al. (2019b) for a more detailed report of musicianship measures. All participants gave informed consent and were paid 300 Danish kroner (approximately

TABLE 1 Participants' demographic and musical expertise information

\begin{tabular}{lll} 
& Musicians & Nonmusicians \\
\hline Sample size & 20 & 20 \\
\hline Female & 8 & 10 \\
\hline Age & $24.2( \pm 3.09)$ & $26.9( \pm 3.48)$ \\
GMSI & $36.80( \pm 6.77)$ & $10.65( \pm 4.05)$ \\
MET & $84.05( \pm 6.98)$ & $70.00( \pm 9.36)$ \\
\hline
\end{tabular}

Note: Mean and standard deviation are reported. 
40 euro) as compensation. The study was approved by the Regional Ethics Committee (De Videnskabsetiske Komitéer for Region Midtjylland in Denmark) and conducted in accordance with the Helsinki Declaration.

\section{2 | Stimuli}

In the experiment, we included conditions with high-predictability (HP) and low-predictability (LP) stimuli. HP stimuli comprised simple melodies consisting of a four-note repeated pitch pattern that has often been used in musical MMNm paradigms and is known as the Alberti bass (Vuust et al., 2011, 2012; Vuust, Liikala, Näätänen, Brattico, \& Brattico, 2016). LP stimuli consisted of a set of major and minor versions of six novel melodies, which had a much less repetitive internal structure and spanned a broader local pitch range than HP stimuli (Figure 1; see supplementary file 1 in Quiroga-Martinez et al., 2019a for the full stimulus set). Note that here both the pitch alphabet (i.e., the number of possible pitch continuations) and the repetitiveness of the sounds contribute to the (un)predictability of the context, as pitches become harder to anticipate, not only when transition probabilities are less specific, but also when the number of possible continuations is larger. However, the impact of pitch alphabet was minimized by transposing HP melodies to different octaves. Furthermore, in the behavioral experiment reported in QuirogaMartinez et al. (2019a), it was demonstrated that repetitiveness alone affects deviance detection in melodic contexts with equal pitch alphabets.

The predictability of these stimuli was measured in terms of Shannon entropy with IDyOM, a computational model of auditory expectations (Pearce, 2005). Briefly, IDyOM estimates the surprise value (referred to as "information content") of different continuations in a melody based on the probability of melodic patterns that appeared previously in the melody or in a long-term training corpus. Entropy, which is the expected value of surprise, is maximal when all continuations are equally plausible and is minimal when a single continuation is highly likely. The corresponding analyses revealed higher entropy values for the LP than the HP condition (see Quiroga-Martinez et al., 2019a for details).
Individual melodies were 32 notes long, lasted $8 \mathrm{~s}$, and were pseudo-randomly transposed between 0 and 5 semitones upward. The presentation order of the melodies was pseudorandom within each condition. After transposition, the pitch-range of the LP condition spanned 31 semitones from $B 3 \quad\left(F_{0} \approx 247 \mathrm{~Hz}\right)$ to $F 6$ $\left(F_{0} \approx 1,397 \mathrm{~Hz}\right)$. HP melodies were transposed to two different octaves to cover approximately the same pitch range as LP melodies.

For stimulus delivery, a pool of 31 standard piano tones was created with the "Warm-grand" sample in Cubase (Steinberg Media Technology, version 8). Each tone was $250 \mathrm{~ms}$ long, was peakamplitude normalized and had 3-ms-long fade-in and fade-out to prevent clicking. No gaps between tones were introduced. Four types of deviants were pseudo-randomly introduced in the melodies, including pitch, timbre, intensity, and pitch-glide. Pitch deviants were created with Audition (Adobe Systems Incorporated, version 8) by raising the pitch of standard tones by 50 cents. Note that predictability in these stimuli was manipulated only in the pitch dimension by changing pitch-alphabet size and pitch repetitiveness, which resulted in pitch deviants exhibiting the strongest predictability effect in sensor-level analyses; see Quiroga-Martinez, et al. (2019a, 2019b) for further details. Consequently, here we focused exclusively on pitch MMNm responses for the DCM analysis.

Each condition was presented in a separate group of three consecutive blocks, each around $7 \mathrm{~min}$ long (i.e., HP-HP-HP/LP-LP-LP or LP-LP-LP/HP-HP-HP). Within each block, melodies were played one after the other without pauses. At the beginning of each block, a melody with no deviants was added to ensure a certain level of auditory regularity at the outset. One deviant per feature was introduced in each of the 144 melodies per block, amounting to a total of 144 deviants per feature in each condition. The position of each deviant was defined by segmenting the melody into groups of four notes (half a bar in Figure 1), selecting some of these groups, and choosing randomly any of the four places within a group with equal probability. The order of appearance of the different types of deviants was pseudorandom, so that no deviant followed another deviant of the same feature. The selection of four-note groups was counterbalanced among melodies-under the constraints of a combined condition (i.e., melody and bass accompaniment) that was included to assess the predictive processing of simultaneous musical streams (see
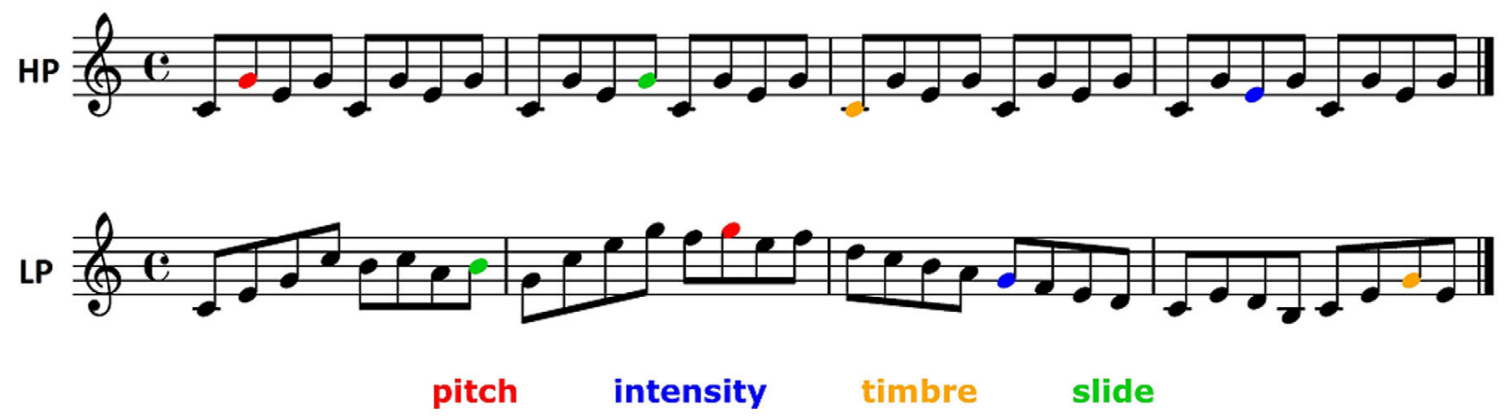

FIGURE 1 Example of the melodies used in the high predictability (HP) and low predictability (LP) conditions. Deviants are indicated with colors. Only pitch deviants were analyzed in this article 
Quiroga-Martinez et al., 2019a, for further details). The analysis of the combined condition is beyond the scope of this article and will be reported elsewhere. LP and HP conditions were counterbalanced across participants and always followed the combined condition.

\section{$2.3 \quad$ Experimental procedure}

Participants received oral and written information, completed musical expertise questionnaires and put on MEG-compatible clothes. We then digitized their head shapes for co-registration with anatomical images and head-position tracking. During the recording, participants were sitting upright in the MEG scanner looking at a screen. Before presenting the musical stimuli, their individual hearing threshold was measured through a staircase procedure using a pure tone with a frequency of $1 \mathrm{kHz}$. The sound level was set at $60 \mathrm{~dB}$ above threshold. We instructed them to watch a silent movie of their choice, ignore the sounds and move as little as possible. Participants were informed there would be musical sequences playing in the background interrupted by short pauses so that they could take a break and adjust their posture. Sounds were presented through isolated MEGcompatible ear tubes (Etymotic ER•30). The recording lasted approximately $90 \mathrm{~min}$, and the whole experimental session took between 2.5 and $3 \mathrm{hr}$, including consent, musical expertise tests, preparation, instructions, breaks, and debriefing.

\section{4 | MEG recording and preprocessing}

Brain magnetic fields were recorded with an Elekta Neuromag MEG TRIUX system with 306 channels (204 planar gradiometers and 102 magnetometers) and a sampling rate of $1,000 \mathrm{~Hz}$. Continuous head position information (cHPI) was obtained with four coils attached to the forehead and the mastoids. Offline, the temporal extension of the signal source separation (tSSS) technique (Taulu \& Simola, 2006) was used to isolate signals coming from inside the skull employing Elekta's MaxFilter software (Version 2.2.15). This procedure included movement compensation for all participants except two nonmusicians, for whom continuous head position information was not reliable due to suboptimal placement of the coils. These participants, however, evinced reliable auditory event-related fields (ERFs), as verified by visual inspection of the amplitude and polarity of the $\mathrm{P} 5 \mathrm{O}(\mathrm{m})$ component. Electrocardiography, electrooculography, and independent component analysis were used to correct for eye-blink and heartbeat artifacts, employing a semiautomatic routine (FastICA algorithm and functions "find_bads_eog" and "find_bads_ecg" in MNE-Python; Gramfort et al., 2013). Visual inspection of the rejected components served as a quality check.

Using the Fieldtrip toolbox (version r9093) (Oostenveld, Fries, Maris, \& Schoffelen, 2011) in MATLAB (R2016a, The MathWorks Inc.), epochs comprising a time window of $400 \mathrm{~ms}$ after sound onset were extracted and baseline-corrected with a prestimulus baseline of $100 \mathrm{~ms}$. Epochs were then low-pass-filtered with a cut-off frequency of $35 \mathrm{~Hz}$ and down-sampled to a resolution of $256 \mathrm{~Hz}$. For each participant, ERFs were computed by averaging the responses for the deviants and averaging a matched selection of an equal number of standards. The evoked responses were then converted to SPM format for the source localization and DCM analyses. Sensor-level statistical analyses of these data have been reported in Quiroga-Martinez, et al. (2019a, 2019b) and Quiroga-Martinez, et al. (2020). Given that we used the same preprocessed data reported in our previous studies-where planar gradiometers were combined by taking the root mean square of channel pairs-in this article, we restricted our analyses to magnetometer data, as in Quiroga-Martinez, et al. (2020).

\subsection{Source localization and network structure}

To identify the auditory networks underlying the processing of pitch MMNm responses, we localized the neural generators of the standard and deviant evoked responses. For this, we used Multiple Sparse Priors (Friston et al., 2008) implemented in SPM12 (version 7478). T1- and T2-weighted magnetic resonance anatomical images (MRI) were obtained-in a separate session for each participant-with a magnetization-prepared two rapid gradient echo (MP2RAGE) sequence (Marques et al., 2010) in a Siemens Magnetom Skyra 3T scanner. The two images were then combined, segmented, projected into $\mathrm{MNI}$ coordinates, and automatically co-registered with the MEG sensor array using digitized head shapes and preauricular and nasion landmarks. We verified the quality of the co-registrations by visual inspection. Lead fields were computed using a single-shell boundary element model with 20,484 dipoles (fine grid). For each participant, we extracted a volume reflecting the mean inverse solution from 0 to $300 \mathrm{~ms}$ after sound onset. The resulting images were then submitted to a group-level repeated-measures ANOVA with deviance (standard vs. deviant) and predictability (HP vs. LP) as factors. No distinction between musicians and nonmusicians was made in these analyses. The resulting statistical parametric maps were corrected for multiple comparisons with random field theory and revealed a clear effect of deviance on source strength. There were two clusters (Figure 2), one in each hemisphere, encompassing five peaks: left (IA1, $x=-50, y=-16, z=-4)$ and right Heschl's gyrus (rA1; $x=46, y=-16, z=0$ ), left (ISTG; $x=-58$, $y=-6, z=6$ ) and right (rSTG; $x=56, y=2, z=-1$ ) anterior STG, and rFOP $(x=50, y=4, z=12)$. The coordinates of these peaks were used as spatial priors for the five nodes or sources of our DCM network.

Note that this network is very similar to classical DCM studies of MMN responses (Dietz, Friston, Mattingley, Roepstorff, \& Garrido, 2014; Garrido et al., 2008; Garrido, Kilner, Kiebel, et al., 2009; Schmidt et al., 2013) with two important differences. First, we include anterior STG (instead of posterior STG or planum temporale), which has been related to the processing of pitch sequences (Gander et al., 2019; Patterson, Uppenkamp, Johnsrude, \& Griffiths, 2002). Second, we include the FOP, which is posterior to the inferior frontal gyrus node used previously. To determine whether the source estimates in the FOP node were an artifact of source leakage, we evaluated the evidence for models with and without this node using Bayesian model comparison. 
(a) Source of the MMN and network structure

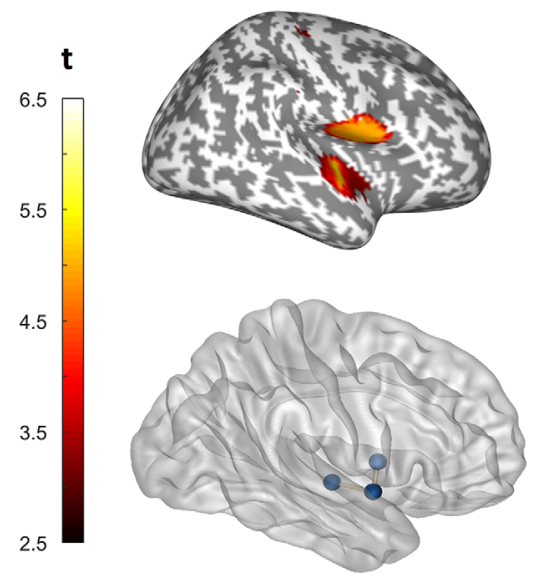

(b) Scheme of network structure

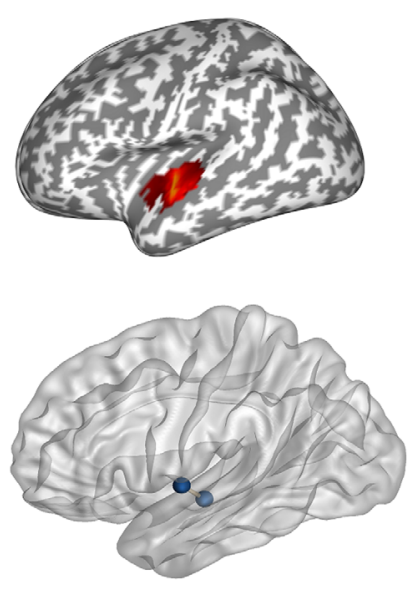

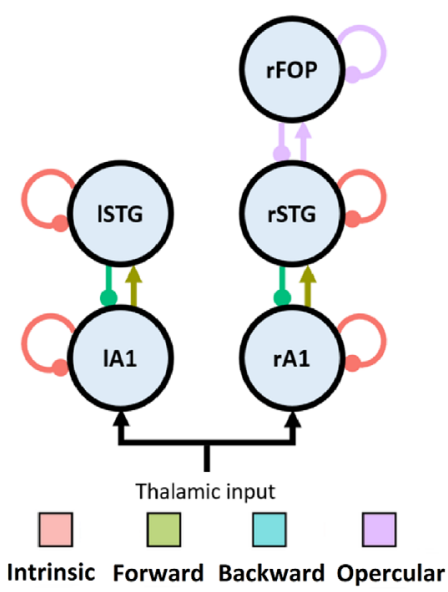

FIGURE 2 Structure of the network. (a) Results of the source reconstruction (top) and prior location of the sources (bottom). (b) Scheme of the network. The connections modulated in each of the four model families are indicated with colors. The combination of these families yielded a total of $2^{4}=16$ models, including a null model in which no connections were modulated. A1, primary auditory cortex; FOP, frontal operculum; STG, superior temporal gyrus

\section{6 | Dynamic causal modeling}

The aim of DCM (Friston, Harrison, \& Penny, 2003; Moran, Pinotsis, \& Friston, 2013) is to assess the evidence for different hypotheses about how observed neuronal activity is generated. These hypotheses are specified through a generative model in which the activity of neuronal populations evolves dynamically according to the structure and (state or condition dependent) connectivity of the network. To estimate the model parameters characterizing directed (effective) synaptic efficacy the predicted neuronal responses are projected (through a lead field) to sensor space for comparison with the recorded data, in an iterative process that employs Bayesian inference (David et al., 2006). This furnishes a posterior distribution over all model parameters (i.e., effective connectivity and condition-specific changes) and the marginal likelihood of the data in the form of a variational free energy bound on model evidence.

The generative model used here is based on the canonical microcircuit (Bastos et al., 2012), which includes four populations of neurons: Spiny stellate cells, inhibitory interneurons, and superficial and deep pyramidal cells (Figure 3). Within a brain region or node (denoted by $i$ ), intrinsic communication between these populations results in overall inhibition. Between region, extrinsic excitatory forward connections project from superficial pyramidal cells in lower areas to spiny cells and deep pyramidal cells in higher areas, whereas extrinsic inhibitory backward connections project from deep pyramidal cells in higher areas, to superficial pyramidal cells and inhibitory interneurons in lower areas. Note that some excitatory connections result in inhibition of their targets, mediated by intermediate inhibitory synapses not modeled explicitly.

The dynamics of the network are given by the set of coupled differential equations shown in Figure 3. Here, the vector $x$ represents the input current (even subscripts) and voltage (odd subscripts) of the corresponding populations of neurons. The matrices $A$ and $G$ respectively define the extrinsic and intrinsic connection strengths between neuronal populations. The time constants $\tau$ define the rate of change of the neuronal dynamics, whereas $\sigma$ is a sigmoid activation function transforming voltages into firing rates. It is assumed that spiny cells receive input from lower regions denoted by $\mathbf{u}$. The o operator denotes element-wise product.

\subsection{1 | Model structure and comparisons}

We defined a network comprising five sources: rA1, IA1, rSTG, ISTG, and rFOP. Forward connections were set from A1 to STG and from STG to FOP, whereas backward connections were set from FOP to STG and from STG to A1. The A1 nodes received simulated auditory thalamic input modeled with a temporal Gaussian bump function, peaking at $60 \mathrm{~ms}$ after sound onset. We used point dipoles to model each source. The prior location of each dipole was obtained from the source localization estimates above and the dipole orientation and location were optimized during model fitting. We assumed interhemispheric dipole symmetry, as warranted by the bilateral auditory generators.

In a first level (within subject) DCM analysis, we modeled the average evoked response from 0 to $300 \mathrm{~ms}$ after sound onset, separately for each of the two predictability conditions. We defined the standard sound as the baseline (reflected in the default connectivity matrix A) and allowed certain connections to change during processing of the deviant sound (as defined in a connectivity matrix B). Note that B-parameters effectively encode the difference between standards and deviants and, therefore, the modulation of effective connectivity that underwrites the MMN response. By switching on and off different $B$ parameters, we were able to 


$$
\begin{aligned}
& \dot{x}_{1}=x_{2} \quad \text { spiny stellate cells } \\
& \dot{x}_{2}=\frac{1}{\tau_{1}} \circ\left(\mathbf{A}_{1} \sigma\left(x_{3}\right)+\mathbf{u}-\mathbf{G}_{1} \circ \sigma\left(x_{1}\right)-\mathbf{G}_{3} \circ \sigma\left(x_{5}\right)-\mathbf{G}_{2} \circ \sigma\left(x_{3}\right)-2 x_{2}-\frac{1}{\tau_{1}} \circ x_{1}\right) \\
& \dot{x}_{3}=x_{4} \quad \text { superficial pyramidal cells } \\
& \dot{x}_{4}=\frac{1}{\tau_{2}} \circ\left(-\mathbf{A}_{3} \sigma\left(x_{7}\right)+\mathbf{G}_{8} \circ \sigma\left(x_{1}\right)-\mathbf{G}_{7} \circ \sigma\left(x_{3}\right)-2 x_{4}-\frac{1}{\tau_{2}} \circ x_{3}\right) \\
& \dot{x}_{5}=x_{6} \quad \text { inhibitory interneurons } \\
& \dot{x}_{6}=\frac{1}{\tau_{3}} \circ\left(-\mathbf{A}_{4} \sigma\left(x_{7}\right)+\mathbf{G}_{5} \circ \sigma\left(x_{1}\right)+\mathbf{G}_{6} \circ \sigma\left(x_{7}\right)-\mathbf{G}_{4} \circ \sigma\left(x_{5}\right)-2 x_{6}-\frac{1}{\tau_{3}} \circ x_{5}\right) \\
& \dot{x}_{7}=x_{8} \quad \text { deep pyramidal cells } \\
& \dot{x}_{8}=\frac{1}{\tau_{4}} \circ\left(\mathbf{A}_{2} \sigma\left(x_{3}\right)-\mathbf{G}_{10} \circ \sigma\left(x_{7}\right)-\mathbf{G}_{9} \circ \sigma\left(x_{5}\right)-2 x_{8}-\frac{1}{\tau_{4}} \circ \boldsymbol{x}_{7}\right)
\end{aligned}
$$

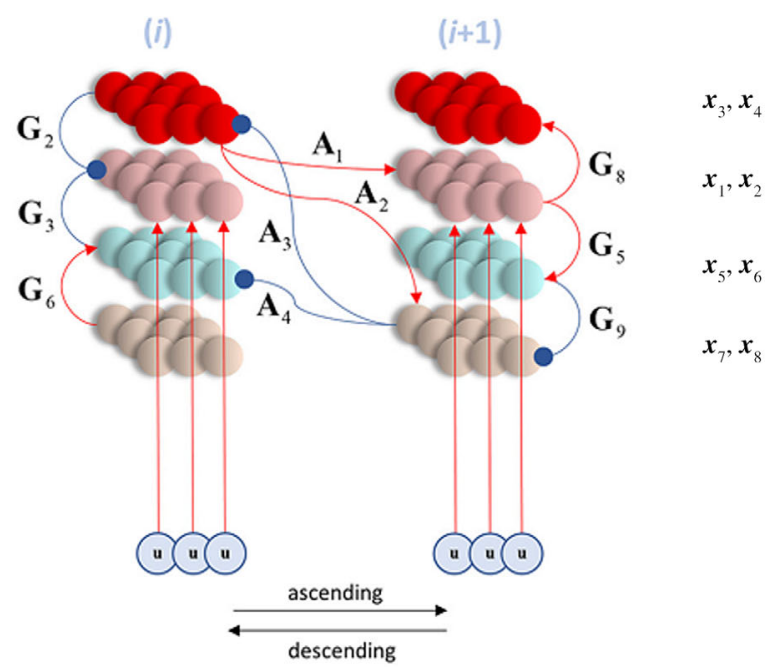

FIGURE 3 State equations (left) and generative model (right) based on the canonical microcircuit (Bastos et al., 2012). The activity of four populations of neurons (indicated by the $\mathbf{x}$ vectors and marked with different colors) evolves according to a set of coupled differential equations defined by the excitatory (red arrows) and inhibitory (blue arrows) connections in the network and a set of intrinsic (matrix G) and extrinsic (matrix A) synaptic weights. Extrinsic synaptic weights pertain to connections between brain regions or nodes, here denoted by $i$. See main text for further details. Figure taken with permission from Parr, Mirza, Cagnan, and Friston (2019)

generate and test different hypotheses about how $\mathrm{MMN}$ responses are generated.

Thus, our model space included modulations of forward and backward connections between A1 and STG, modulations of intrinsic connections in A1 and STG, and different combinations of these. We also included models in which connections within, to, and from the FOP were switched on and off-to assess whether its contribution to source estimates was due to leakage from temporal areas, or whether it participated in the network dynamics. By switching on and off each of these families, we ended up with $2^{4}=16$ models: 15 grouped in the families intrinsic, forward, backward and FOP (Figure $2 \mathrm{~b}$ ), and a null model in which no connections were modulated. Note that the families are not mutually exclusive such that a given model could belong to more than one family. For each participant and condition, we fitted the full model, which encompassed the modulation of all the B-parameters.

\subsection{2 | Group-level analyses}

First-level B-parameter estimates were submitted to a second level (between subject or group) analysis using Parametric Empirical Bayes (PEB), a technique in which the group-level variance is used as a hyperprior to constrain the random effects on first-level parameters (Zeidman et al., 2019). The second level model was a general linear model (GLM) that enabled us to assess the evidence that (a specific set of) B-parameters were modulated by stimulus deviance, melodic predictability, musicianship, or the predictability-by-musicianship interaction. The parameters of the second level model constitute an interaction between each factor of the GLM and each B-parameter of a DCM: for example, the effect of melodic predictability on the change in intrinsic connectivity within left $\mathrm{A} 1$.
All factors in the GLM (i.e., design matrix) were mean-centered. To test our hypotheses, we assessed the evidence that the above factors had an effect on the B-parameters, by comparing the evidence for all models in which the factor is switched on, with that of all models in which it is switched off (including the null model). Note that an effect of stimulus deviance is simply a nonzero B-parameter, that is modeled with the constant term in the GLM. The above analysis was repeated for a series of planned comparisons for each family of first level DCMs, that is, prespecified hypotheses about the connections mediating the mismatch responses.

In a complementary analysis, we used Bayesian model averaging to estimate the effects parameterized by the second level model, that is, the posterior density over model parameters weighted by the posterior probability of the models considered. Finally, in an exploratory analysis, we used Bayesian model reduction (Friston et al., 2016), which performs a "greedy" search over all possible models-including those outside our hypothesis space-by beginning with a full model that includes all the above effects, then pruning away redundant parameters (that are not necessary to account for the data and just add to model complexity). The (log) probability that a B-parameter is modulated by a factor is given as the difference in log evidence between second level models with and without the effect in question.

\section{3 | RESULTS}

\subsection{Effective connectivity underlying the MMN}

Compared to standard sounds, deviant sounds reduced the inhibitory intrinsic connections in A1 and STG and inhibitory backward connections from STG to A1. This is reflected in the high posterior probabilities (>.90) of "backward" and "intrinsic" families (Figure 4a, first 

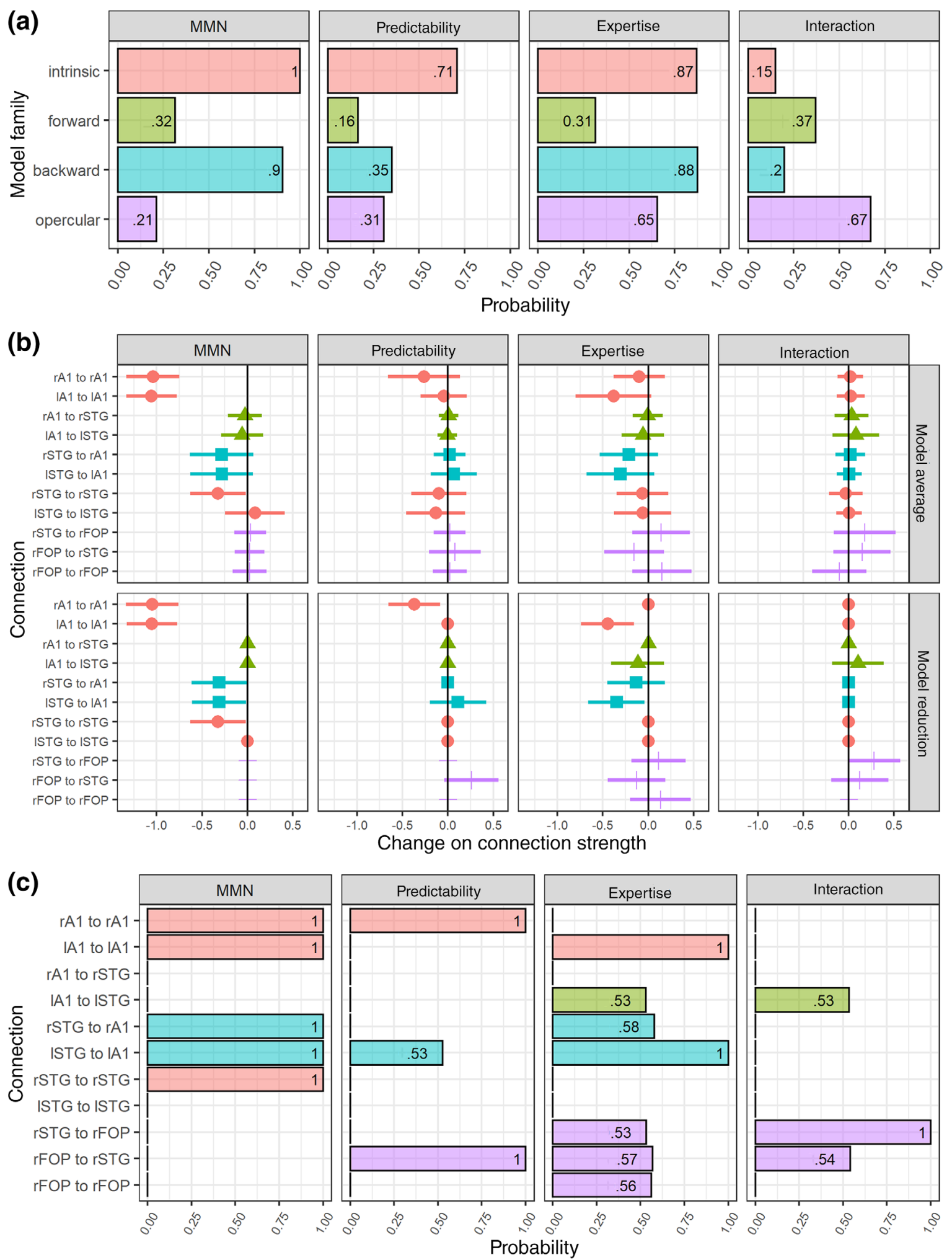

Model family: $=$ Intrinsic $\rightleftharpoons$ Forward $=$ Backward + Opercular

FIGURE 4 Results of group-level DCM analyses. (a) Posterior probability of the modulation of B-parameters in each family. Note that families are not mutually exclusive and therefore the sum of their probabilities may exceed 1. (b) Parameter estimates after Bayesian model reduction (top) and averaging (bottom), corresponding to the modulation of connection strengths by sound deviance (MMN), melodic predictability, musical expertise, and the predictability-by-expertise interaction. In model averaging, parameter values are weighted by the posterior probability of the models in the hypotheses space. In contrast, in exploratory model reduction, the evidence for parametric effects is obtained from an exhaustive search across all possible model configurations, in which effects with little evidence are pruned away (i.e., set to 0). Error bars represent $95 \%$ credible intervals. (c) Posterior probability of the modulation of each parameter after Bayesian model reduction 
column). In contrast, the "forward" family has a much lower posterior probability (.32). The family-wise analysis also showed a low posterior probability for the "opercular" family, suggesting that modulations of frontal opercular activity are unlikely to explain the MMN responses observed in this experiment.

The results of Bayesian model averaging, and Bayesian model reduction were largely consistent with the results of the planned Bayesian family comparisons. 95\% credible intervals excluded zero in the case of intrinsic connections ( $\mathrm{rA1}$, IA1, and rSTG) for both model averaging and reduction, and in the case of backward connections (rSTG to rA1 and ISTG to IA1) for model reduction only (Figure 4b, first column). Furthermore, intrinsic, and backward parameters had high posterior probabilities (>.99) after Bayesian model reduction (Figure 4c, first column). The modulation of these connections by stimulus deviance implies a disinhibition and a corresponding increase in the excitability or gain, of $\mathrm{A} 1$ and $\mathrm{rSTG}$ contributions to the MMN.

To assess the accuracy of the DCM results, we compared the observed sensor-level data with those predicted by the model. Figures 5 and 6 show that the predicted data match the observed data well, reproducing the topography of magnetic fields and the larger responses for predictable compared to less predictable melodies and for musicians compared to nonmusicians, especially in the left hemisphere.

\subsection{Effect of melodic predictability}

We found reduced intrinsic connectivity in rA1 for HP compared to LP melodies, as shown by the relatively high probability of the "intrinsic" family (.71) (Figure 4a, second column). In contrast, the posterior probabilities of forward (.16), backward (.25), and opercular (.31) families were much lower. The rA1 parameter had a high probability $(>.99$ Figure $4 \mathrm{c}$, second column) and its corresponding $95 \%$ credible interval excluded 0 (Figure 4b, second column) after Bayesian model reduction. Furthermore, the exploratory model reduction also indicated the strengthening of the connection from rFOP to rSTG, as shown by the high probability of this effect (>.99, Figure $4 c$, second column). However, this was not reflected in the probability of the opercular family (Figure $4 \mathrm{a}$, second column), so suggesting that this modulation was highly specific to the backward opercular connection within the right hemisphere and did not reflect a general contribution of the FOP.

\subsection{Effect of musical expertise}

We observed a decrease in the strength of intrinsic and backward inhibitory connections-that is, disinhibition-in musicians compared to nonmusicians, as shown in the high probability of "intrinsic" (.87) and "backward" (.88) families (Figure 4a, third column). Posterior probabilities for forward (.31) and opercular (.65) families were low and moderate. $95 \%$ credible intervals excluded 0 in the case of the intrinsic IA1 connection for both model reduction and averaging, and in the case of the backward ISTG to IA1 connection for model reduction only (Figure $4 \mathrm{~b}$, third column). Moreover, the probabilities of these two parameters were high after model reduction (>.99, Figure 4c, third column).

\subsection{Interaction between predictability and expertise}

We did not observe strong evidence for a predictability-by-expertise interaction, as reflected in the low posterior probabilities of intrinsic (.15), forward (.37), and backward (.20) families (Figure 4a, fourth column). However, the probability of the opercular family was moderate (.67) and exploratory model reduction suggested a strengthening in the connection from rSTG to rFOP for musicians in the predictable

Observed
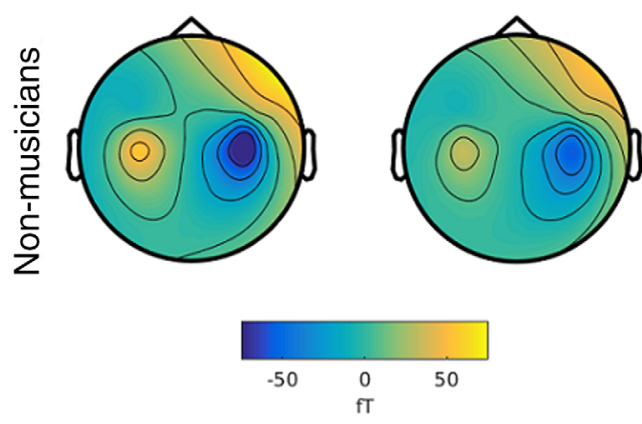

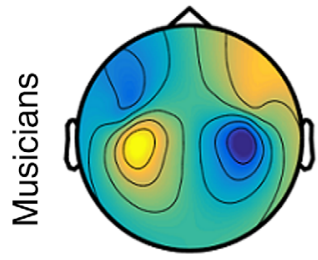

HP

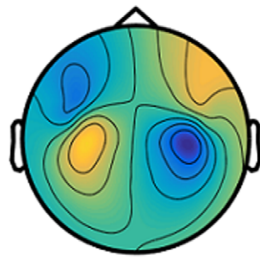

LP
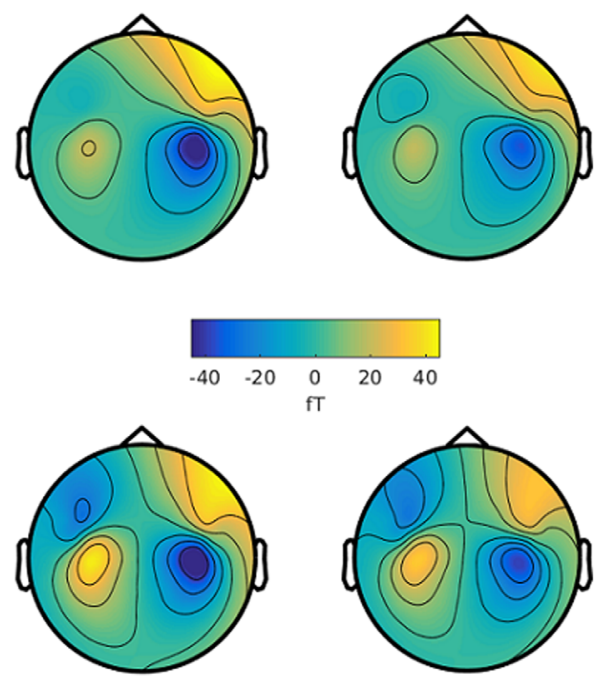

LP
FIGURE 5 Topography of grand average $\mathrm{MMNm}$ responses (difference between deviants and standards) from 170 to $210 \mathrm{~ms}$ after sound onset, as observed in the experiment and predicted by DCM. HP, high predictability, LP, low predictability 

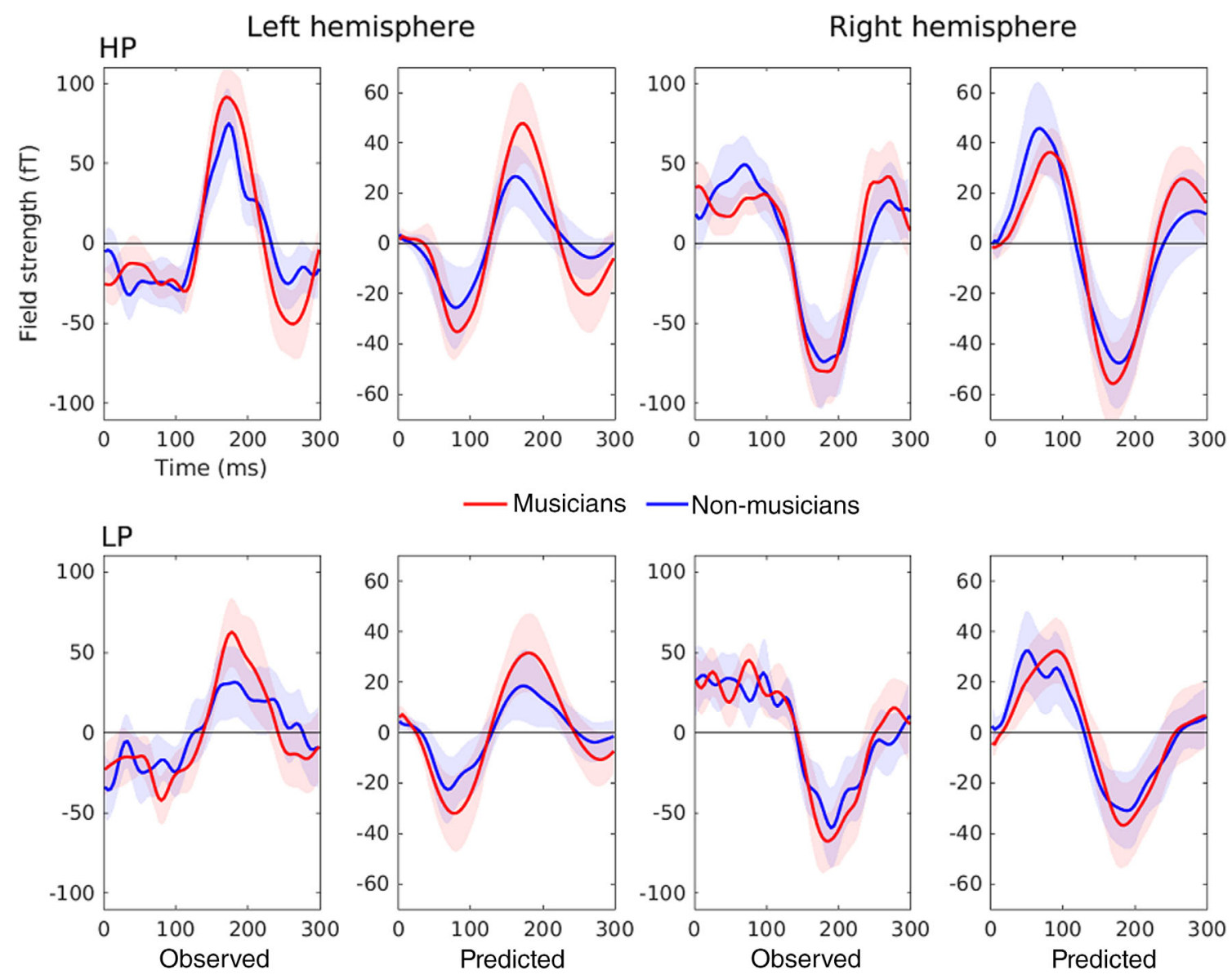

FIGURE 6 Event-related field of the MMNm (difference between deviants and standards) for each condition, group, and hemisphere, as observed in the experiment and predicted by DCM. The time courses correspond to the average of representative left (1611, 1621, 0231 , and 0241) and right $(2411,2421,1331$, and 1341$)$ auditory channels. Shaded areas depict $95 \%$ confidence intervals. HP, high predictability, LP, low predictability. Note that, while data were originally baseline corrected, for DCM they were mean centered as shown in the figure

condition. For this parameter, the credible interval excluded 0 (Figure $4 \mathrm{~b}$, fourth column, bottom panel) and the posterior probability was high (>.99, Figure 4c, fourth column).

\section{4 | DISCUSSION}

In this study, we found that the MMN responses elicited by surprising sounds in music listening-and the effects of predictability and expertise on these responses-rest on disinhibition of auditory areas, as indicated by reduced intrinsic connectivity within A1 and STG and backward connectivity from STG to A1. This supports the notion that neural gain, as a plausible mechanism for mediating precisionweighted prediction error, underlies the salience of surprising sounds and the strength of the neural responses they generate.

\subsection{Connectivity patterns underlying the MMN}

The main contributors to the MMN were modulations of intrinsic connectivity within bilateral $\mathrm{A} 1$ and $\mathrm{rSTG}$, and bilateral backward connectivity from STG to A1. Reduced intrinsic connectivity implies an increase in the excitability of neural populations and has been interpreted as a salience-related enhancement of neural gain in response to deviant sounds (Auksztulewicz et al., 2017, 2018; Auksztulewicz \& Friston, 2015). In other words, mistuned sounds may attract attentional resources and thus be prioritized at the earliest stages of auditory cortical processing.

That we found a reduction in top-down inhibition from secondary to primary auditory areas and a lack of modulation of forward connections contrasts with most previous studies in which both forward and backward connections show oddball-related effects (Auksztulewicz \& Friston, 2015; Chennu et al., 2016; Garrido, Kilner, Kiebel, \& Friston, 2007; Garrido et al., 2008; Garrido, Kilner, Kiebel, et al., 2009; Lumaca, Dietz, Hansen, Quiroga-Martinez, \& Vuust, 2020; Schmidt et al., 2013). From a predictive coding perspective (Friston, 2005; Garrido, Kilner, Stephan, et al., 2009; Huang \& Rao, 2011), forward and backward communication between brain areas reflects the update of predictive models by prediction error. Thus, the reason why backward connections were weakened, and forward connections remained unchanged might be that out-of-tune deviants (which violate a rather low-level musical regularity) are handled locally in the auditory cortex 
and elicit little model updating (i.e., learning) at higher stages where melodic expectations are likely processed. This, in turn, might indicate that out-of-tune sounds are heard as occasional, attention-grabbing "wrong" notes, rather than structurally novel events demanding a change in the current predictive model of the melody. In other words, a tone in a melody could still be predicted and recognized, even when it is saliently out-of-tune. A similar view has been proposed by Koelsch, Vuust, and Friston (2018), who suggest that, in typical musical MMN designs, the higher-order predictive model is so strong that deviant sounds elicit prediction error responses that do not get resolved at higher processing levels. As the authors put it, deviant sounds "fall on deaf ears" and "keep knocking on the door" (p. 6).

Another finding that differs from previous research is the lack of involvement of frontal areas in the generation of the MMN, as indicated by the low probability of the opercular family. This is consistent with the lack of frontal generators previously reported for the same dataset (Quiroga-Martinez et al., 2019b) and in a recent fMRI study using simple musical stimuli (Lumaca et al., 2020). This suggests that the opercular peak found in the present source-level statistical analyses may reflect source leakage. Thus, the acoustic deviations introduced (i.e., out-of-tune sounds) may have been resolved at low-level processing stages in the temporal lobe, without engaging frontal areas typically involved in the sequential processing of sounds (Koelsch et al., 2002, 2009). This may have been reinforced by the fact that participants were instructed to ignore the sounds and watch a film instead.

Interestingly, an EEG study using the same stimuli as here found that $\mathrm{MMN}$ responses were similarly modulated by melodic predictability in participants with congenital amusia (a condition that disrupts pitch processing) and controls (Quiroga-Martinez et al., 2021). Since amusia most likely arises from reduced connectivity between temporal and frontal areas (Albouy et al., 2013; Peretz, 2016), this further indicates that the processes underlying the mistuning $M M N$ and its modulation by predictability and musicianship are restricted to local auditory areas in the temporal lobe. Note, however, that exploratory Bayesian model reduction indicated that connections between rFOP and rSTG may have been modulated by predictability and the predictability-by-expertise interaction. This could indicate that, as sounds become more salient, higher-order brain areas are engaged. However, further research is needed to properly assess this claim and dissociate it from source leakage.

\section{2 | Enhancement of neural gain in predictable melodies}

The strength of intrinsic (inhibitory) connections in rA1 was reduced in predictable compared to less predictable melodies. Such connectivity changes may thus underlie the stronger MMN response observed for the former. This is consistent with the hypothesis that the stimulus-driven increase in predictive precision enhances the sensitivity to upcoming sensory signals, thus providing evidence for the role of gain modulation in precision weighting of prediction error. This effect was found only in the right hemisphere, which may reflect the fact that musical pitch processing in the general population is predominantly right-lateralized (Albouy, Benjamin, Morillon, \& Zatorre, 2020; Zatorre, Belin, \& Penhune, 2002).

\section{3 | Left-lateralized gain enhancement in musicians}

Compared to nonmusicians, musicians showed disinhibition in IA1 and backward connections from ISTG to IA1. This indicates that the stronger $M M N$ response in this group might rest on the same gain enhancement mechanism found for the effect of predictability. In turn, this suggests that both phenomena could be framed as enhancements in predictive precision. In previous work, we have proposed the terms "stimulus-driven" and "expertise-driven" to refer to these two types of uncertainty reduction (Quiroga-Martinez et al., 2019b). Thus, here we show that, although they seem to affect prediction error responses independently, stimulus-driven, and expertise-driven uncertainty reduction might rely on similar underlying changes in effective connectivity. Furthermore, these results agree with the enhanced responses previously found in musicians for violations of pitch-related regularities such as interval, contour, musical tuning, and pitch patterns (Boh, Herholz, Lappe, \& Pantev, 2011; Fujioka, Trainor, Ross, Kakigi, \& Pantev, 2004; Herholz, Lappe, \& Pantev, 2009; Koelsch, Schröger, \& Tervaniemi, 1999; Tervaniemi et al., 2014; Vuust et al., 2012).

Interestingly, the expertise-related gain enhancement was left lateralized, which adds to a collection of left-hemisphere specific effects linked to musical expertise (Ellis, Bruijn, Norton, Winner, \& Schlaug, 2013; Elmer, Hänggi, Meyer, \& Jäncke, 2013; Limb, Kemeny, Ortigoza, Rouhani, \& Braun, 2006; Ono et al., 2011; Tervaniemi, Sannemann, Noyranen, Salonen, \& Pihko, 2011; Vuust et al., 2005). Considering the hemispheric specialization for temporal versus spectral information and for music versus language (Albouy et al., 2020; Zatorre et al., 2002), this could mean that musicians' pitch processing involves a finer temporal evaluation of the sounds and more explicit lexical knowledge of musical structure.

\subsection{A plausible mechanism for precision- weighted prediction error}

Taken together, our results suggest that the modulation of gain in auditory areas may underlie the weighting of prediction error responses by uncertainty (Clark, 2013; Feldman \& Friston, 2010; Hohwy, 2012), where uncertainty corresponds to unpredictable melodic contexts and a lack of musical expertise in the current experimental design. Effects on intrinsic connectivity are not necessarily explained by short or long-term synaptic plasticity, but rather by modulations of synaptic efficacy through acetylcholine or other classical neuromodulatory neurotransmitters (Auksztulewicz \& Friston, 2016; Baldeweg et al., 2006). Changes in synaptic efficacy may also be 
mediated by fast synchronous interactions, involving spiking inhibitory interneurons equipped with NMDA receptors (Schmidt et al., 2013). Consistent with our results, the precision weighting of prediction errors has been cast as reflecting unexpected uncertainty-that is, a momentary change in the estimated predictability of the context induced by unexpected events-which has been associated with modulations of pupil diameter and the neuromodulator norepinephrine (Bianco, Ptasczynski, \& Omigie, 2020; Dayan \& Yu, 2006; Zhao et al., 2019). Thus, a plausible hypothesis is that the enhanced excitability of auditory cortex in response to deviant sounds has its origins in neuromodulation-and concomitant changes in synchronous gain. Note, however, that we also found evidence for a reduction of backward (inhibitory) connectivity, suggesting that the observed effects were, at least in part, mediated by changes in the sensitivity to topdown afferents from cortical sources higher in the auditory hierarchy. These changes, nonetheless, are quantitatively smaller than those in intrinsic connections and further contribute to the disinhibition of A1, thereby facilitating gain enhancement. Future research should aim to disentangle the specific contribution of neuromodulation and changes in synaptic efficacy to gain control in A1.

\section{5 | CONCLUSION}

In this study, we characterized the neuronal dynamics and changes in synaptic efficacy underlying the salience of pitch deviants and its modulation by melody predictability and musical expertise during music listening. Using DCM, we found that musicianship and predictability, as well as deviance itself, increased neural gain in primary auditory cortex through a reduction in the strength of intrinsic (inhibitory) connectivity in A1 and STG. The MMN effect was also associated with reduced backward connectivity from STG to A1. Gain modulation in primary auditory cortex was right-lateralized in the case of predictability and left-lateralized in the case of musical expertise. Our findings are consistent with predictive processing theories suggesting that precise and informative error signals are prioritized by the brain for subsequent hierarchical processing. Furthermore, they suggest that the ability to contextualize sensory processing in musicianship and predictable sensory streams relies on similar neuronal gain mechanisms.

\section{ACKNOWLEDGMENTS}

The authors wish to thank the project initiation group, namely Christopher Bailey, Torben Lund, and Dora Grauballe, for their help with setting up the experiments. The authors also thank Nader Sedghi, Massimo Lumaca, Giulia Donati, Ulrika Varankaite, Giulio Carraturo, Riccardo Proietti, and Claudia lorio for assistance during MEG recordings. The Center for Music in the Brain is funded by the Danish National Research Foundation (DNRF 117). Emma Holmes is funded by Action on Hearing Loss (PA_25). Niels Christian Hansen received funding from the European Union's Horizon 2020 research and innovation programme under the Marie Skłodowska-Curie grant agreement No. 754513 and The Aarhus Universitets Forskningsfond. Karl
Friston was funded by a Wellcome Trust Principal Research Fellowship (Ref: 088130/Z/09/Z). The funders had no influence on the scientific content of this article.

\section{CONFLICT OF INTEREST}

The authors report no conflict of interest.

\section{AUTHOR CONTRIBUTIONS}

David R. Quiroga-Martinez: Conceptualization; methodology; software; formal analysis; data curation; writing-original draft; visualization; investigation. Niels Christian Hansen: Conceptualization; methodology; and writing-original draft. Andreas Højlund: Conceptualization; methodology; software; and writing-original draft; supervision. Marcus Pearce: Software; formal analysis; writing-original draft. Emma Holmes: Supervision and writing-original draft. Elvira Brattico: Conceptualization; supervision; writing-original draft. Karl Friston: Supervision; Writing-original draft. Peter Vuust: Conceptualization; methodology; supervision; writing-original draft; and funding acquisition.

\section{DATA AVAILABILITY STATEMENT}

Data sharing is not applicable to this article as no new data were created or analyzed in this study.

\section{ORCID}

David R. Quiroga-Martinez (D) https://orcid.org/0000-0001-8242-

6395

Karl Friston (D) https://orcid.org/0000-0001-7984-8909

\section{REFERENCES}

Albouy, P., Benjamin, L., Morillon, B., \& Zatorre, R. J. (2020). Distinct sensitivity to spectrotemporal modulation supports brain asymmetry for speech and melody. Science, 367(6481), 1043-1047. https://doi.org/ 10.1126/science.aaz3468

Albouy, P., Mattout, J., Bouet, R., Maby, E., Sanchez, G., Aguera, P.-E., ... Tillmann, B. (2013). Impaired pitch perception and memory in congenital amusia: The deficit starts in the auditory cortex. Brain, 136(5), 1639-1661. https://doi.org/10.1093/brain/awt082

Auksztulewicz, R., Barascud, N., Cooray, G., Nobre, A. C., Chait, M., \& Friston, K. J. (2017). The cumulative effects of predictability on synaptic gain in the auditory processing stream. The Journal of Neuroscience, 37(28), 6751-6760. https://doi.org/10.1523/JNEUROSCI.0291-17. 2017

Auksztulewicz, R., \& Friston, K. J. (2015). Attentional enhancement of auditory mismatch responses: A DCM/MEG study. Cerebral Cortex, 25(11), 4273-4283. https://doi.org/10.1093/cercor/bhu323

Auksztulewicz, R., \& Friston, K. J. (2016). Repetition suppression and its contextual determinants in predictive coding. Cortex, 80, 125-140. https://doi.org/10.1016/j.cortex.2015.11.024

Auksztulewicz, R., Schwiedrzik, C. M., Thesen, T., Doyle, W., Devinsky, O., Nobre, A. C., ... Melloni, L. (2018). Not all predictions are equal: "What" and "when" predictions modulate activity in auditory cortex through different mechanisms. Journal of Neuroscience, 38(40), 86808693. https://doi.org/10.1523/JNEUROSCI.0369-18.2018

Baldeweg, T., Wong, D., \& Stephan, K. (2006). Nicotinic modulation of human auditory sensory memory: Evidence from mismatch negativity potentials. International Journal of Psychophysiology, 59(1), 49-58. https://doi.org/10.1016/j.ijpsycho.2005.07.014 
Bastos, A. M., Usrey, W. M., Adams, R. A., Mangun, G. R., Fries, P., \& Friston, K. J. (2012). Canonical microcircuits for predictive coding. Neuron, 76(4), 695-711. https://doi.org/10.1016/j.neuron.2012.10.038

Bianco, R., Ptasczynski, L. E., \& Omigie, D. (2020). Pupil responses to pitch deviants reflect predictability of melodic sequences. Brain and Cognition, 138, 103621. https://doi.org/10.1016/j.bandc.2019.103621

Boh, B., Herholz, S. C., Lappe, C., \& Pantev, C. (2011). Processing of complex auditory patterns in musicians and nonmusicians. PLoS One, 6(7), e21458. https://doi.org/10.1371/journal.pone.0021458

Chennu, S., Noreika, V., Gueorguiev, D., Shtyrov, Y., Bekinschtein, T. A., \& Henson, R. (2016). Silent expectations: Dynamic causal modeling of cortical prediction and attention to sounds that weren't. Journal of Neuroscience, 36(32), 8305-8316. https://doi.org/10.1523/ JNEUROSCI.1125-16.2016

Clark, A. (2013). Whatever next? Predictive brains, situated agents, and the future of cognitive science. Behavioral and Brain Sciences, 36(03), 181-204. https://doi.org/10.1017/S0140525X12000477

David, O., Kiebel, S. J., Harrison, L. M., Mattout, J., Kilner, J. M., \& Friston, K. J. (2006). Dynamic causal modeling of evoked responses in EEG and MEG. Neurolmage, 30(4), 1255-1272. https://doi.org/10. 1016/j.neuroimage.2005.10.045

Dayan, P., \& Yu, A. J. (2006). Phasic norepinephrine: A neural interrupt signal for unexpected events. Network: Computation in Neural Systems, 17(4), 335-350. https://doi.org/10.1080/09548980601004024

Dietz, M. J., Friston, K. J., Mattingley, J. B., Roepstorff, A., \& Garrido, M. I. (2014). Effective connectivity reveals right-hemisphere dominance in Audiospatial perception: Implications for models of spatial neglect. The Journal of Neuroscience, 34(14), 5003-5011. https://doi.org/10.1523/ JNEUROSCI.3765-13.2014

Ellis, R. J., Bruijn, B., Norton, A. C., Winner, E., \& Schlaug, G. (2013). Training-mediated leftward asymmetries during music processing: A crosssectional and longitudinal fMRI analysis. Neurolmage, 75, 97-107. https://doi.org/10.1016/j.neuroimage.2013.02.045

Elmer, S., Hänggi, J., Meyer, M., \& Jäncke, L. (2013). Increased cortical surface area of the left planum temporale in musicians facilitates the categorization of phonetic and temporal speech sounds. Cortex, 49(10), 2812-2821. https://doi.org/10.1016/j.cortex.2013.03.007

Feldman, H., \& Friston, K. J. (2010). Attention, uncertainty, and freeenergy. Frontiers in Human Neuroscience, 4, 215. https://doi.org/10. 3389/fnhum.2010.00215

Friston, K. J. (2005). A theory of cortical responses. Philosophical Transactions of the Royal Society B: Biological Sciences, 360(1456), 815-836. https://doi.org/10.1098/rstb.2005.1622

Friston, K. J., Harrison, L., Daunizeau, J., Kiebel, S., Phillips, C., TrujilloBarreto, N., ... Mattout, J. (2008). Multiple sparse priors for the M/EEG inverse problem. Neurolmage, 39(3), 1104-1120. https://doi. org/10.1016/j.neuroimage.2007.09.048

Friston, K. J., Harrison, L., \& Penny, W. (2003). Dynamic causal modelling. Neurolmage, 19(4), 1273-1302. https://doi.org/10.1016/S1053-8119 (03)00202-7

Friston, K. J., Litvak, V., Oswal, A., Razi, A., Stephan, K. E., van Wijk, B. C. M., ... Zeidman, P. (2016). Bayesian model reduction and empirical Bayes for group (DCM) studies. Neurolmage, 128, 413-431. https://doi.org/10.1016/j.neuroimage.2015.11.015

Friston, K. J., Sajid, N., Quiroga-Martinez, D. R., Parr, T., Price, C. J., \& Holmes, E. (2020). Active listening. Hearing Research, 399, 107998. https://doi.org/10.1016/j.heares.2020.107998

Fujioka, T., Trainor, L. J., Ross, B., Kakigi, R., \& Pantev, C. (2004). Musical training enhances automatic encoding of melodic contour and interval structure. Journal of Cognitive Neuroscience, 16(6), 1010-1021. https://doi.org/10.1162/0898929041502706

Gander, P. E., Kumar, S., Sedley, W., Nourski, K. V., Oya, H., Kovach, C. K., ... Griffiths, T. D. (2019). Direct electrophysiological mapping of human pitch-related processing in auditory cortex. Neurolmage, 202, 116076. https://doi.org/10.1016/j.neuroimage.2019.116076

Garrido, M. I., Friston, K. J., Kiebel, S. J., Stephan, K. E., Baldeweg, T., \& Kilner, J. M. (2008). The functional anatomy of the MMN: A DCM study of the roving paradigm. Neurolmage, 42(2), 936-944. https:// doi.org/10.1016/j.neuroimage.2008.05.018

Garrido, M. I., Kilner, J. M., Kiebel, S. J., \& Friston, K. J. (2007). Evoked brain responses are generated by feedback loops. Proceedings of the National Academy of Sciences, 104(52), 20961-20966. https://doi.org/ 10.1073/pnas.0706274105

Garrido, M. I., Kilner, J. M., Kiebel, S. J., \& Friston, K. J. (2009). Dynamic causal modeling of the response to frequency deviants. Journal of Neurophysiology, 101(5), 2620-2631. https://doi.org/10.1152/jn.90291. 2008

Garrido, M. I., Kilner, J. M., Stephan, K. E., \& Friston, K. J. (2009). The mismatch negativity: A review of underlying mechanisms. Clinical Neurophysiology, 120(3), 453-463. https://doi.org/10.1016/j.clinph.2008. 11.029

Garrido, M. I., Rowe, E. G., Halász, V., \& Mattingley, J. B. (2018). Bayesian mapping reveals that attention boosts neural responses to predicted and unpredicted stimuli. Cerebral Cortex, 28(5), 1771-1782. https:// doi.org/10.1093/cercor/bhx087

Garrido, M. I., Sahani, M., \& Dolan, R. J. (2013). Outlier responses reflect sensitivity to statistical structure in the human brain. PLoS Computational Biology, 9(3), e1002999. https://doi.org/10.1371/journal.pcbi. 1002999

Gramfort, A., Luessi, M., Larson, E., Engemann, D., Strohmeier, D., Brodbeck, C., ... Hämäläinen, M. (2013). MEG and EEG data analysis with MNE-python. Frontiers in Neuroscience, 7, 267. https://doi.org/ 10.3389/fnins.2013.00267

Hansen, N. C., \& Pearce, M. (2014). Predictive uncertainty in auditory sequence processing. Frontiers in Psychology, 5, 1052. https://doi.org/ 10.3389/fpsyg.2014.01052

Hansen, N. C., Vuust, P., \& Pearce, M. (2016). "If you have to ask, you'll never know": Effects of specialised stylistic expertise on predictive processing of music. PLoS One, 11(10), e0163584. https://doi.org/10. 1371/journal.pone.0163584

Heilbron, M., \& Chait, M. (2018). Great expectations: Is there evidence for predictive coding in auditory cortex? Neuroscience, 389, 54-73. https://doi.org/10.1016/j.neuroscience.2017.07.061

Herholz, S. C., Lappe, C., \& Pantev, C. (2009). Looking for a pattern: An MEG study on the abstract mismatch negativity in musicians and nonmusicians. BMC Neuroscience, 10(1), 42. https://doi.org/10.1186/ 1471-2202-10-42

Hohwy, J. (2012). Attention and conscious perception in the hypothesis testing brain. Frontiers in Psychology, 3, 96. https://doi.org/10.3389/ fpsyg.2012.00096

Hsu, Y.-F., Bars, S. L., Hämäläinen, J. A., \& Waszak, F. (2015). Distinctive representation of Mispredicted and unpredicted prediction errors in human electroencephalography. Journal of Neuroscience, 35(43), 14653-14660. https://doi.org/10.1523/JNEUROSCI.2204-15.2015

Huang, Y., \& Rao, R. P. N. (2011). Predictive coding. Wiley Interdisciplinary Reviews: Cognitive Science, 2(5), 580-593. https://doi.org/10.1002/ wcs.142

Kliuchko, M., Brattico, E., Gold, B. P., Tervaniemi, M., Bogert, B., Toiviainen, P., \& Vuust, P. (2019). Fractionating auditory priors: A neural dissociation between active and passive experience of musical sounds. PLoS One, 14(5), e0216499. https://doi.org/10.1371/journal. pone.0216499

Koelsch, S., Gunter, T. C., Cramon, D. Y. V., Zysset, S., Lohmann, G., \& Friederici, A. D. (2002). Bach speaks: A cortical "language-network" serves the processing of music. Neurolmage, 17(2), 956-966. https:// doi.org/10.1006/nimg.2002.1154 
Koelsch, S., Schröger, E., \& Tervaniemi, M. (1999). Superior pre-attentive auditory processing in musicians. Neuroreport, 10(6), 1309-1313. https://doi.org/10.1097/00001756-199904260-00029

Koelsch, S., Schulze, K., Sammler, D., Fritz, T., Müller, K., \& Gruber, O. (2009). Functional architecture of verbal and tonal working memory: An FMRI study. Human Brain Mapping, 30(3), 859-873. https://doi. org/10.1002/hbm.20550

Koelsch, S., Vuust, P., \& Friston, K. J. (2018). Predictive processes and the peculiar case of music. Trends in Cognitive Sciences, 23, 63-77. https:// doi.org/10.1016/j.tics.2018.10.006

Limb, C. J., Kemeny, S., Ortigoza, E. B., Rouhani, S., \& Braun, A. R. (2006). Left hemispheric lateralization of brain activity during passive rhythm perception in musicians. The Anatomical Record Part A: Discoveries in Molecular, Cellular, and Evolutionary Biology, 288A(4), 382-389. https://doi.org/10.1002/ar.a.20298

Lumaca, M., Dietz, M. J., Hansen, N., Quiroga-Martinez, D. R., \& Vuust, P. (2020). Perceptual learning of tone patterns changes the effective connectivity between Heschl's gyrus and planum temporale. Human Brain Mapping, 42, 941-952. https://doi.org/10.1002/hbm.25269

Marques, J. P., Kober, T., Krueger, G., van der Zwaag, W., Van de Moortele, P.-F., \& Gruetter, R. (2010). MP2RAGE, a self bias-field corrected sequence for improved segmentation and T1-mapping at high field. Neurolmage, 49(2), 1271-1281. https://doi.org/10.1016/j. neuroimage.2009.10.002

Moran, R. J., Pinotsis, D. A., \& Friston, K. J. (2013). Neural masses and fields in dynamic causal modeling. Frontiers in Computational Neuroscience, 7, 57. https://doi.org/10.3389/fncom.2013.00057

Müllensiefen, D., Gingras, B., Musil, J., \& Stewart, L. (2014). The musicality of non-musicians: An index for assessing musical sophistication in the general population. PLoS One, 9(2), e89642. https://doi.org/10.1371/ journal.pone.0089642

Ono, K., Nakamura, A., Yoshiyama, K., Kinkori, T., Bundo, M., Kato, T., \& Ito, K. (2011). The effect of musical experience on hemispheric lateralization in musical feature processing. Neuroscience Letters, 496(2), 141145. https://doi.org/10.1016/j.neulet.2011.04.002

Oostenveld, R., Fries, P., Maris, E., \& Schoffelen, J.-M. (2011). FieldTrip: Open source software for advanced analysis of MEG, EEG, and invasive electrophysiological data. Computational Intelligence and Neuroscience, 2011, 1-9. https://doi.org/10.1155/2011/156869

Parr, T., Mirza, M. B., Cagnan, H., \& Friston, K. J. (2019). Dynamic causal modelling of active vision. Journal of Neuroscience, 39(32), 6265-6275. https://doi.org/10.1523/JNEUROSCI.2459-18.2019

Patterson, R. D., Uppenkamp, S., Johnsrude, I. S., \& Griffiths, T. D. (2002). The processing of temporal pitch and melody information in auditory cortex. Neuron, 36(4), 767-776. https://doi.org/10.1016/S0896-6273 (02)01060-7

Pearce, M. (2005). The construction and evaluation of statistical models of melodic structure in music perception and composition. London: City University.

Peretz, I. (2016). Neurobiology of congenital Amusia. Trends in Cognitive Sciences, 20(11), 857-867. https://doi.org/10.1016/j.tics.2016.09.002

Quiroga-Martinez, D. R., Hansen, N. C., Højlund, A., Pearce, M., Brattico, E., \& Vuust, P. (2020). Decomposing neural responses to melodic surprise in musicians and non-musicians: Evidence for a hierarchy of predictions in the auditory system. Neurolmage, 215, 116816. https://doi.org/10.1016/j.neuroimage.2020.116816

Quiroga-Martinez, D. R., Hansen, N. C., Højlund, A., Pearce, M., Brattico, E., \& Vuust, P. (2019a). Reduced prediction error responses in high-as compared to low-uncertainty musical contexts. Cortex, 120, 181-200. https://doi.org/10.1016/j.cortex.2019.06.010

Quiroga-Martinez, D. R., Hansen, N. C., Højlund, A., Pearce, M., Brattico, E., \& Vuust, P. (2019b). Musical prediction error responses similarly reduced by predictive uncertainty in musicians and non-musicians. European Journal of Neuroscience, 51, 2250-2269. https://doi. org/10.1111/ejn.14667
Quiroga-Martinez, D. R., Tillmann, B., Brattico, E., Cholvy, F., Fornoni, L., Vuust, P., \& Caclin, A. (2021). Listeners with congenital amusia are sensitive to context uncertainty in melodic sequences. Neuropsychologia, 158, 107911. http://doi.org/10.1016/j.neuropsychologia.2021.107911

Rabinowitz, N. C., Goris, R. L., Cohen, M., \& Simoncelli, E. P. (2015). Attention stabilizes the shared gain of V4 populations. elife, 4, e08998. https://doi.org/10.7554/eLife.08998

Reynolds, J. H., \& Desimone, R. (1999). The role of neural mechanisms of attention in solving the binding problem. Neuron, 24(1), 19-29. https://doi.org/10.1016/S0896-6273(00)80819-3

Ross, S., \& Hansen, N. C. (2016). Dissociating prediction failure: Considerations from music perception. Journal of Neuroscience, 36(11), 3103-3105. https://doi.org/10.1523/JNEUROSCI.0053-16. 2016

Schmidt, A., Diaconescu, A. O., Kometer, M., Friston, K. J., Stephan, K. E., \& Vollenweider, F. X. (2013). Modeling ketamine effects on synaptic plasticity during the mismatch negativity. Cerebral Cortex, 23(10), 23942406. https://doi.org/10.1093/cercor/bhs238

Southwell, R., \& Chait, M. (2018). Enhanced deviant responses in patterned relative to random sound sequences. Cortex, 109, 92-103. https://doi.org/10.1016/j.cortex.2018.08.032

Taulu, S., \& Simola, J. (2006). Spatiotemporal signal space separation method for rejecting nearby interference in MEG measurements. Physics in Medicine and Biology, 51(7), 1759-1768. https://doi.org/10. 1088/0031-9155/51/7/008

Tervaniemi, M., Huotilainen, M., \& Brattico, E. (2014). Melodic multifeature paradigm reveals auditory profiles in music-sound encoding. Frontiers in Human Neuroscience, 8, 496. https://doi.org/10.3389/ fnhum.2014.00496

Tervaniemi, M., Sannemann, C., Noyranen, M., Salonen, J., \& Pihko, E. (2011). Importance of the left auditory areas in chord discrimination in music experts as demonstrated by MEG: Importance of the left auditory areas in chord discrimination. European Journal of Neuroscience, 34(3), 517-523. https://doi.org/10.1111/j.1460-9568.2011. 07765.x

Vuust, P., Brattico, E., Glerean, E., Seppänen, M., Pakarinen, S., Tervaniemi, M., \& Näätänen, R. (2011). New fast mismatch negativity paradigm for determining the neural prerequisites for musical ability. Cortex, 47(9), 1091-1098. https://doi.org/10.1016/j.cortex.2011.04. 026

Vuust, P., Brattico, E., Seppänen, M., Näätänen, R., \& Tervaniemi, M. (2012). Practiced musical style shapes auditory skills. Annals of the new York Academy of Sciences, 1252(1), 139-146. https://doi.org/10.1111/ j.1749-6632.2011.06409.x

Vuust, P., Dietz, M. J., Witek, M., \& Kringelbach, M. L. (2018). Now you hear it: A predictive coding model for understanding rhythmic incongruity. Annals of the New York Academy of Sciences, 1423(1), 19-29. https://doi.org/10.1111/nyas.13622

Vuust, P., Liikala, L., Näätänen, R., Brattico, P., \& Brattico, E. (2016). Comprehensive auditory discrimination profiles recorded with a fast parametric musical multi-feature mismatch negativity paradigm. Clinical Neurophysiology, 127(4), 2065-2077. https://doi.org/10.1016/j.clinph. 2015.11.009

Vuust, P., Pallesen, K. J., Bailey, C., van Zuijen, T. L., Gjedde, A., Roepstorff, A., \& Østergaard, L. (2005). To musicians, the message is in the meter: Pre-attentive neuronal responses to incongruent rhythm are left-lateralized in musicians. Neurolmage, 24(2), 560-564. https:// doi.org/10.1016/j.neuroimage.2004.08.039

Wallentin, M., Højlund, A., Friis-Olivarius, M., Vuust, C., \& Vuust, P. (2010). The musical ear test, a new reliable test for measuring musical competence. Learning and Individual Differences, 20(3), 188-196. https://doi. org/10.1016/j.lindif.2010.02.004

Zatorre, R. J., Belin, P., \& Penhune, V. B. (2002). Structure and function of auditory cortex: Music and speech. Trends in Cognitive Sciences, 6(1), 37-46. https://doi.org/10.1016/S1364-6613(00)01816-7 
Zeidman, P., Jafarian, A., Seghier, M. L., Litvak, V., Cagnan, H., Price, C. J., \& Friston, K. J. (2019). A guide to group effective connectivity analysis, part 2: Second level analysis with PEB. Neurolmage, 200, 12-25. https://doi.org/10.1016/j.neuroimage. 2019.06.032

Zhao, S., Chait, M., Dick, F., Dayan, P., Furukawa, S., \& Liao, H.-I. (2019). Pupil-linked phasic arousal evoked by violation but not emergence of regularity within rapid sound sequences. Nature Communications, 10(1), 4030. https://doi.org/10.1038/s41467-01912048-1
How to cite this article: Quiroga-Martinez, D. R., Hansen, N. C., Højlund, A., Pearce, M., Brattico, E., Holmes, E., Friston, K., \& Vuust, P. (2021). Musicianship and melodic predictability enhance neural gain in auditory cortex during pitch deviance detection. Human Brain Mapping, 42(17), 5595-5608. https:// doi.org/10.1002/hbm.25638 